\title{
ÉTUDE DE L'ANGUILLE EN MARAIS D'EAU DOUCE. PREMIÉRE ANALYSE DES PROBABILITÉS DE CAPTURE OBSERVÉES LORS DES INVENTAIRES PAR PÊCHE ÉLECTRIQUE.
}

\author{
P. LAMBERT (1), E. FEUNTEUN (2) et C. RIGAUD (1)
}

(1) CEMAGREF de Bordeaux, Division Aquaculture et Pêche, 50 avenue de Verdun, BP 3, 33611 Gazinet Cedex, France.

(2) FISH-PASS, 91 rue de Saint-Brieuc, 35000 Rennes, France.

\section{RÉSUMÉ}

A partir du résultat de 28 inventaires ( 3 passages) par pêche électrique réalisés dans des canaux de petites dimensions du Marais Breton, une analyse des captures d'anguilles (541 individus) est effectuée.

Dans un tiers des stations, deux passages se révèlent insuffisants pour parvenir à une estimation quantitative satisfaisante ; celle-ci s'avère, en effet, inférieure à la somme des captures réalisées sur l'ensemble des 3 passages.

Sur 3 passages, les méthodes d'estimation basées sur une probabilité de capture (Pc) constante ne conviennent pas dans $20 \%$ des stations et conduisent à une surestimation des effectifs les plus probables (augmentation de Pc au cours des passages). L'usage de méthodes permettant de tester des variations de Pc au cours des passages présente alors beaucoup d'intérêt, l'une d'entre elles avec variante créée par nos soins a été mise en oeuvre au cours de ce travail.

Enfin, toutes stations confondues, aucune relation générale entre la taille des anguilles et leur probabilité de capture n'a été mise en évidence. L'examen, au niveau de chaque station, laisse toutefois supposer une influence des caractéristiques du milieu sur l'évolution de Pc en fonction de la taille.

Mots-clés : Anguilla anguilla, marais endigué, canaux, pêche électrique, méthodologie.

EEL STUDY IN FRESHWATER MARSHES.

FIRST ANALYSIS OF CATCH PROBABILITY OBSERVED DURING ELECTRIC FISHING OPERATIONS.

\section{SUMMARY}

Twenty-eight stations in shallow ditches were sampled with electricity ( 3 fishings) in a freshwater marsh of the French atlantic coast (Marais breton). Eel catches (541 eels) are analysed.

Removal methods, assuming the catch probability $(P c)$ remains constant with successive fishings, can't be used in nearly $20 \%$ of the cases and would give an overestimation of the most probable number of eels in these stations ( $\mathrm{Pc}$ increasing). 
On one third of the stations, only two successive fishings are insufficient for accurate quantitative assessment. In fact, on these stations, the stock assessment is lower than total catches with three fishings.

So, it seemed interesting to apply an existing assessment method, with one original development, allowing a Pc variation with successive fishings.

Among all stations, no general relation between eel size and Pc variations is revealed. However, further analysis must be engaged to explain some variations revealed within stations.

Key-words : Anguilla anguilla, freshwater marshes, electric fishing, methodology.

\section{INTRODUCTION}

La quantification des stocks d'anguilles présents dans les réseaux de fossés et de canaux des marais d'eau douce du littoral atlantique est un élément important pouvant déboucher à terme sur des plans raisonnés d'exploitation et de gestion de l'espèce dans ces territoires particulièrement bien situés au sein de son aire de répartition.

Cet objectif nécessite la mise en oeuvre d'inventaires à grande échelle sur ces réseaux très denses de fossés offrant toute une mosaïque d'habitats disponibles (FEUNTEUN et al., 1992).

Il y a déjà quelques années, le "Groupe national Anguille» (ANONYME, 1984) avait souligné les importantes lacunes existant dans le domaine des méthodes d'échantillonnage et d'estimation des stocks dans les divers milieux aquatiques continentaux. Les marais littoraux n'échappent bien sûr pas à ce constat.

Dans le cas de cours d'eau ou canaux de petites dimensions, la pêche électrique apparaît comme un outil particulièrement à même de déboucher rapidement sur des estimations quantitatives spécifiques dans le cadre d'inventaires avec passages successifs sans remise sur secteurs isolés.

Néammoins, il est maintenant de plus en plus admis que cet outil d'échantillonnage présente comme les autres un caractère sélectif (BACKIEL, 1990) qu'il convient d'analyser et de préciser.

Outre les hypothèses d'absence d'immigration, d'émigration, de mortalité et de natalité lors des opérations, la quasi-totalité des méthodes d'estimation des effectifs les plus probables (DE LURY, 1947 ; MORAN, 1951 ; ZIPPIN, 1956) sur une station échantillonnée par passages successifs sans remise repose sur celles d'équiprobabilité de capture entre les individus et de constance de cette probabilité de capture $(\mathrm{Pc})$ au cours des divers passages (COWX, 1983 ; GERDEAUX, 1987 ; BOHLIN, 1990).

Actuellement, on emploie le plus fréquemment la méthode de CARLE et STRUB (1978) qui autorise une variation a priori de Pc entre les différents passages. Cette connaissance a priori étant peu fréquente, l'application habituelle repose sur la constance de Pc d'un passage à l'autre (GERDEAUX, 1987 ; FEUNTEUN 1994).

Or, au travers du résultat de diverses études menées en particulier sur des cyprinidés et salmonidés, cette hypothèse de constance de Pc semble devoir être sérieusement reconsidérée (BOHLIN et COWX, 1990 ; LIBOSVARSKY, 1990). et relèvent :

Les causes de variation de Pc sont d'ailleurs multiples (ZALEWSKI et COWX, 1990)

— soit de considérations théoriques :

des différences de probabilité individuelle de capture (notamment en fonction de la taille) entraînent une décroissance de Pc d'un passage à l'autre (BOHLIN et COWX, 1990),

- soit de considérations comportementales: 
- la tendance à se cacher après un premier choc électrique et/ou une sensibilité accrue lors d'un deuxième stimulus électrique (LELEK in LIBOSVARSKY, 1990) conduisent à une diminution de Pc,

- Pc peut également augmenter si une pêche électrique a chassé un certain nombre de poissons hors de leurs cachettes inaccessibles lors des passages précédents (SCHNUTE, 1983), phénomène évoqué dans le cas de l'anguille par NAISMITH et KNIGHTS (1990).

Enfin, les protocoles de pêche électrique les plus souvent appliqués ne permettent pas de standardiser réellement l'effort de pêche d'un passage à l'autre. La variabilité de Pc s'en trouve d'autant plus accrue.

SCHNUTE (1983), en faisant appel à des algorithmes peiformants de recherche de minimum de vraisemblance, développe une méthodologie permettant de tester, outre le modèle classique avec probabilité de capture constante, des modèles avec variation de ce facteur d'un passage à l'autre.

Nous avons donc choisi d'utiliser sa méthode de traitement des informations sur un jeu de données issues de campagnes de pêche électrique dans le marais breton entre 1987 et 1990 pour analyser, sur l'anguille, le niveau de variabilité de Pc en fonction de la taille des individus ( 541 individus de 8 à $76 \mathrm{cms}$ ) et du rang du passage considéré.

\section{MATÉRIELS ET MÉTHODES}

\section{Matériels}

Les différents traitements ont été réalisés sur un jeu de 28 stations inventoriées avec 3 passages successifs (tableau I) au cours d'une étude sur le marais de Bourgneuf, marais littoral situé à l'ouest de la France (FEUNTEUN et al., 1992 ; FEUNTEUN, 1994).

Cet hydrosystème, constitué d'un réseau extrêmement dense de fossés de faibles profondeurs (gabarits moyens d'environ $90 \mathrm{cms}$ ) et de faibles largeurs (moyenne d'environ 3 mètres), se caractérise par la grande hétérogénéité des conditions de milieu (niveau de colonisation par les végétaux flottants ou fixés, niveau de colmatage,...).

Chaque station d'inventaire a été délimitée par des filets droits à maille carrée de 5 $\mathrm{mm}$ de côté placés de 30 à 50 mètres de distance, la longueur minimale d'une station d'échantillonnage étant de 30 mètres (FEUNTEUN, 1987).

Les pêches électriques étaient effectuées à l'aide du matériel “Héron" dont les caractéristiques sont décrites par LAMARQUE et al. (1978). Une anode circulaire de 50 centimètres de diamètre, montée sur une perche, était manipulée depuis un zodiac, luimême manoeuvré depuis les rives par deux opérateurs grâce à des cordages. Au cours de la période d'étude, nous avons utilisé un courant continu de 7-10 A et de 250-300 V pour une conductivité comprise entre 500 et $1600 \mu \mathrm{s} \mathrm{cm}^{-1}$ (moyenne $578 \mu \mathrm{sm}^{-1}$ ).

Les opérations ont été réalisées de manière à standardiser l'effort de pêche entre les stations et au sein d'une même station au cours des passages successifs (maintien de la même équipe de pêche, constance du temps de pêche).

\section{Méthode d'estimation statistique des effectifs}

Pour l'estimation de l'effectif le plus probable sur une station donnée, la méthode utilisée est celle mise au point par SCHNUTE en 1983. Elle présente l'avantage de tester statistiquement plusieurs modèles dont certains offrent des possibilités de variation de la probabilité de capture.

\section{Ont ainsi été mis en oeuvre dans cette étude :}

- le premier modèle de Schnute (modèle1) repose, comme la méthode de CARLE et STRUB (1978), sur l'hypothèse d'une probabilité de capture constante au cours des passages successifs, 
Tableau I : Effectifs capturés lors des différents passages et somme des individus capturés au bout de trois passages.

Table I : Eel catches during the three fishings and total catches.

\begin{tabular}{crrrr}
\hline station & C1 & C2 & C3 & \multicolumn{1}{c}{$T$} \\
\hline B081-8705 & 20 & 15 & 4 & 39 \\
B081-8806 & 10 & 8 & 7 & 25 \\
B083-8906 & 19 & 8 & 8 & 35 \\
B083-8909 & 14 & 0 & 2 & 16 \\
C021-8705 & 11 & 7 & 3 & 21 \\
C021-8806 & 11 & 10 & 4 & 25 \\
D131-8707 & 18 & 9 & 3 & 30 \\
D131-8806 & 10 & 6 & 0 & 16 \\
D131-8906 & 5 & 2 & 0 & 7 \\
D131-9003 & 7 & 1 & 0 & 8 \\
D132-8806 & 7 & 5 & 2 & 14 \\
D132-8909 & 4 & 1 & 0 & 5 \\
D133-8707 & 10 & 7 & 6 & 23 \\
D133-8806 & 21 & 20 & 9 & 50 \\
D133-8906 & 8 & 10 & 4 & 22 \\
D133-8909 & 12 & 6 & 4 & 22 \\
D134-8906 & 11 & 2 & 0 & 13 \\
D134-9003 & 5 & 2 & 1 & 8 \\
G062-8806 & 6 & 5 & 2 & 13 \\
G06G-8707 & 19 & 4 & 8 & 31 \\
G06G-8806 & 2 & 4 & 4 & 10 \\
G06G-8909 & 6 & 0 & 1 & 7 \\
H131-8906 & 11 & 3 & 3 & 17 \\
H131-8909 & 6 & 2 & 1 & 9 \\
H131-9003 & 5 & 6 & 1 & 12 \\
H132-8906 & 8 & 4 & 6 & 18 \\
H133-8906 & 12 & 8 & 9 & 29 \\
H134-8906 & 8 & 5 & 3 & 16 \\
\hline & & & &
\end{tabular}

- le deuxième modèle de Schnute (modèle 21) suppose une probabilité de capture constante à partir du deuxième passage,

- une variante du modèle 21, mise au point par nos soins (modèle 22), propose une probabilité de capture constante sur les deux premiers passages et une modification de ce paramètre sur le dernier passage.

Le troisième modèle de Schnute, basé sur une variation monotone de la probabilité de capture au cours des passages successifs, n'a pas été utilisé car son surparamétrage conduit à le rejeter dans la plupart des cas (SCHNUTE, 1983 ; LAMBERT, 1994).

La mise en oeuvre de ces méthodes s'est faite sur le logiciel MATLAB, avec appel à des procédures d'optimisation avec pénalités. Les tests statistiques ont été réalisés grâce au logiciel SYSTAT. 


\section{RÉSULTATS}

\section{Variation de la probabilité de capture en fonction de la taille}

Afin de disposer d'effectifs suffisants dans les divers couples "station - classe de taille "tout en gardant une certaine signification biologique aux classes de taille retenues, le pas de taille a été fixé à $60 \mathrm{~mm}$; ce qui, pour notre échantillon d'anguilles, conduit à l'identification de 8 classes et de 224 couples "station-classe de taille". Seuls les 150 couples présentant des effectifs non nuls ont été pris en compte pour les tests.

L'évolution de la moyenne des probabilités de capture observées dans chaque classe de taille, au premier passage, montre une légère tendance à l'augmentation (figure 1). Toutefois, un test de Kruskal-Wallis ne permet pas de conclure à une différence significative entre les moyennes des différents groupes de taille (test $\mathrm{H} p>.05$ ).

Par contre, ce même test révèle des différences signicatives entre les stations (test $\mathrm{H} \mathrm{p}<.05)$ pour une même classe de taille.

Ces résultats nous ont amenés à ne pas raisonner par classe de taille dans le reste du traitement.

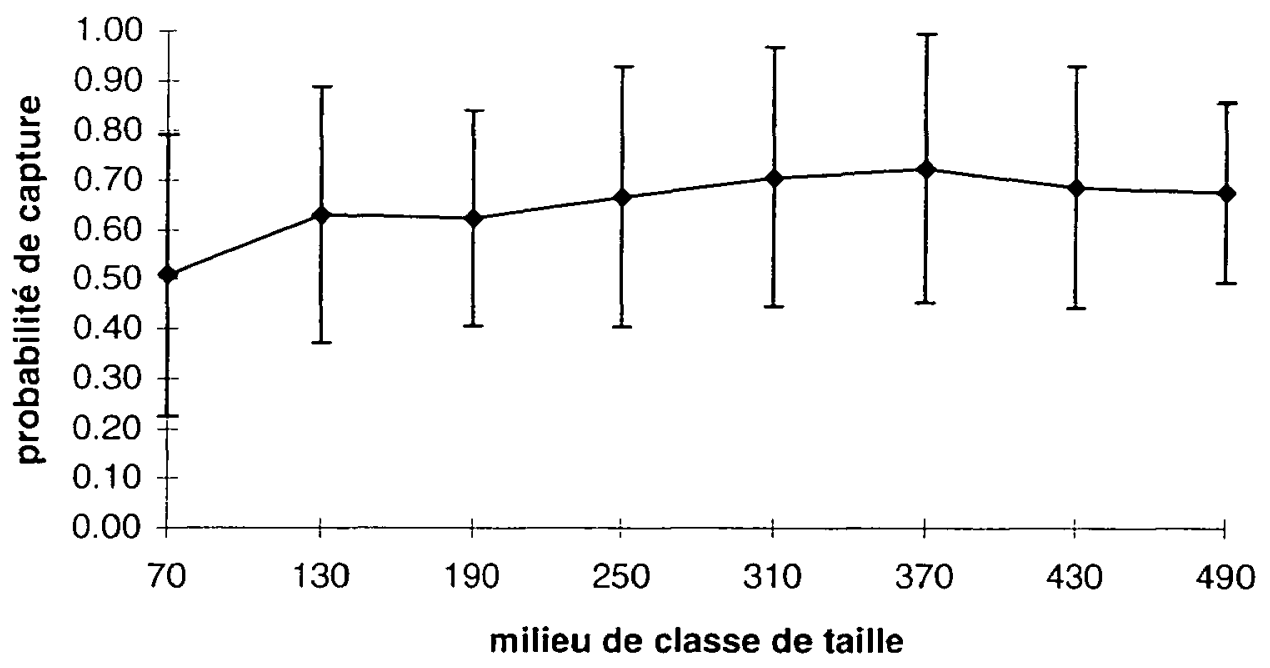

Figure 1 : Évolution (moyennes \pm 2 écarts types) de la probabilité de capture calculée lors du premier passage en fonction des classes de taille.

Figure 1 : Evolution (mean \pm 2 standard deviations) of the capture probability of the first fishing versus length class.

\section{Variation de la probabilité de capture au cours de deux passages}

Au cours du traitement des données obtenues sur deux passages avec recherche et sélection du modèle le plus adapté à chaque inventaire, le modèle 1 de Schnute ( $P C$ constante sur les 2 passages) a été rejeté dans $11 \%$ des cas (tableau II). Sur les trois stations correspondantes, une augmentation de Pc au cours du second passage est enregistrée.

\section{Variation de la probabilité de capture au cours des trois passages}

Au cours du traitement des données obtenues sur trois passages avec recherche et sélection du modèle le plus adapté à chaque inventaire (tableau III), le modèle 1 de Schnute (Pc constante sur tous les passages) a été rejeté dans $18 \%$ des cas ( 5 stations). 
Tableau II : Résultats de 2 modèles de la méthode Schnute en considérant 2 passages.

(en gras, modèle retenu ; $\mathrm{N}$ : effectif estimé, pc : probabilité de capture constante sur les trois passages ; pc1 et pc2 : probabilités de capture au cours respectivement du premier et troisième passage, $F$ : minimum de la fonction de Schnute).

Table II : Results of two Schnute models using two fishings.

(in bold : the best model ; $\mathbf{N}$ : estimate of the initial population, pc : constant catch probability on each fishing, pc1 : catch probability on the first fishing ; pc2 : catch probability on the second fishing, $F$ : minimum of the Schnute function).

\begin{tabular}{|c|c|c|c|c|c|c|c|}
\hline \multirow[b]{3}{*}{ station } & \multicolumn{6}{|c|}{2 passages } & \multirow[b]{3}{*}{$F$} \\
\hline & \multicolumn{3}{|c|}{ Schnute 1} & \multicolumn{3}{|c|}{ Schnute 21} & \\
\hline & $N$ & $\mathrm{pc}$ & $F$ & $N$ & $\mathrm{pc1}$ & $\mathrm{pc} 2$ & \\
\hline B081-8705 & 62.4 & 0.33 & 2.35 & 35.0 & 0.56 & 0.93 & 1.07 \\
\hline B081-8806 & 29.7 & 0.37 & 2.03 & & & & \\
\hline B083-8906 & 30.2 & 0.65 & 1.60 & 27.0 & 0.76 & 0.98 & 0.39 \\
\hline B083-8909 & 14.3 & 0.97 & 1.60 & 17.8 & 0.78 & 0.00 & 1.50 \\
\hline C021-8705 & 23.4 & 0.50 & 1.79 & 18.0 & 0.64 & 0.99 & 0.09 \\
\hline C021-8806 & 47.4 & 0.25 & 2.25 & 21.0 & 0.48 & 0.93 & 0.74 \\
\hline D131-8707 & 32.3 & 0.58 & 1.79 & 27.0 & 0.67 & 1.00 & 0.00 \\
\hline D131-8806 & 19.6 & 0.55 & 1.65 & 16.0 & 0.62 & 1.00 & 0.01 \\
\hline D131-8906 & 7.0 & 0.77 & 0.58 & 7.0 & 0.72 & 0.93 & 0.15 \\
\hline D131-9003 & 8.0 & 0.92 & 0.18 & 8.1 & 0.93 & 0.90 & 0.47 \\
\hline D132-8806 & 15.4 & 0.50 & 1.65 & 12.0 & 0.59 & 1.00 & 0.01 \\
\hline D132-8909 & 5.0 & 0.83 & 0.20 & 5.0 & 0.79 & 0.97 & 0.04 \\
\hline D133-8707 & 23.6 & 0.46 & 1.86 & 17.0 & 0.64 & 1.00 & 0.14 \\
\hline D133-8806 & 160.7 & 0.14 & 2.68 & 41.0 & 0.51 & 1.00 & 0.01 \\
\hline D133-8906 & 167.2 & 0.06 & 2.49 & 18.1 & 0.48 & 0.92 & 0.84 \\
\hline D133-8909 & 18.2 & 0.73 & 1.90 & 18.0 & 0.67 & 1.00 & 0.00 \\
\hline D134-8906 & 13.0 & 0.87 & 0.32 & 13.0 & 0.79 & 0.83 & 0.51 \\
\hline D134-9003 & 7.0 & 0.77 & 0.58 & 7.0 & 0.72 & 0.93 & 0.15 \\
\hline G062-8806 & 15.7 & 0.43 & 1.75 & 11.0 & 0.50 & 0.98 & 0.18 \\
\hline G06G-8707 & 23.2 & 0.84 & 0.72 & 23.0 & 0.85 & 0.88 & 0.57 \\
\hline G06G-8806 & 58.1 & 0.05 & 2.17 & 6.0 & 0.30 & 1.00 & 0.01 \\
\hline G06G-8909 & 6.0 & 1.00 & 0.00 & 7.2 & 0.83 & 0.00 & 0.99 \\
\hline H131-8906 & 14.3 & 0.80 & 0.72 & 14.0 & 0.83 & 0.91 & 0.35 \\
\hline H131-8909 & 8.0 & 0.81 & 0.51 & 8.0 & 0.77 & 0.93 & 0.16 \\
\hline H131-9003 & 35.2 & 0.17 & 2.12 & 11.0 & 0.41 & 0.94 & 0.43 \\
\hline $\mathrm{H} 132-8906$ & 12.9 & 0.68 & 1.25 & 12.0 & 0.67 & 0.97 & 0.13 \\
\hline H133-8906 & 27.6 & 0.46 & 1.912 & 0.0 & 0.64 & 0.98 & 0.22 \\
\hline H134-8906 & 15.6 & 0.56 & 1.56 & 13.0 & 0.66 & 0.97 & 0.21 \\
\hline
\end{tabular}


Tableau III : Résultats de 3 modèles de la méthode Schnute en considérant 3 passages.

(en gras, modèle retenu ; $\mathbf{N}$ : effectif estimé ; pc : probabilité de capture constante sur les trois passages ; pc1 et pc3 : probabilités de capture au cours respectivement du premier et troisième passage ; pc23 et pc12 : probabilités de capture constantes respectivement sur les deux derniers passages et sur les deux premiers passages ; $F$ : minimum de la fonction de Schnute).

Table III : Results of three Schnute models using three fishings.

(in bold : the best model ; $N$ : estimate of the initial population ; pc : constant catch probability on each fishing, pc1 : catch probability on the first fishing ; pc3 : catch probability on the third fishing ; pc12 : constant catch probability on the two first fishings, pc23 : constant catch probability on the two last fishings ; $F$ : minimum of the Schnute function).

\begin{tabular}{|c|c|c|c|c|c|c|c|c|c|c|c|}
\hline \multirow[b]{3}{*}{ station } & \multicolumn{10}{|c|}{3 passages } & \\
\hline & \multicolumn{3}{|c|}{ Schnute 1} & \multicolumn{4}{|c|}{ Schnute 21} & \multicolumn{4}{|c|}{ Schnute 22} \\
\hline & $N$ & pc & $\mathrm{F1}$ & $N$ & pc1 & pc23 & $\mathrm{F} 2$ & $N$ & pc12 & pc3 & $\mathrm{F} 2$ \\
\hline B081-8705 & 43.4 & 0.52 & 2.66 & 39.1 & 0.51 & 0.82 & 0.85 & 58.5 & 0.36 & 0.17 & 2.32 \\
\hline B081-8806 & 44.1 & 0.24 & 2.20 & 34.2 & 0.29 & 0.37 & 2.09 & 25.0 & 0.43 & 0.98 & 0.55 \\
\hline B083-8906 & 42.4 & 0.43 & 2.49 & 50.1 & 0.38 & 0.30 & 2.35 & 35.0 & 0.54 & 0.99 & 0.17 \\
\hline B083-8909 & 16.0 & 0.80 & 3.98 & 16.0 & 0.88 & 0.50 & 2.77 & 16.0 & 0.74 & 0.90 & 3.78 \\
\hline C021-8705 & 23.0 & 0.52 & 1.50 & 21.1 & 0.49 & 0.78 & 0.98 & 21.0 & 0.58 & 0.84 & 0.95 \\
\hline C021-8806 & 30.8 & 0.41 & 2.25 & 25.5 & 0.44 & 0.74 & 1.12 & 25.0 & 0.56 & 0.87 & 2.00 \\
\hline D131-8707 & 30.1 & 0.68 & 1.68 & 30.0 & 0.60 & 0.77 & 0.79 & 30.0 & 0.65 & 0.99 & 0.48 \\
\hline D131-8806 & 16.2 & 0.72 & 2.45 & 16.0 & 0.60 & 0.86 & 0.95 & 18.2 & 0.57 & 0.00 & 1.78 \\
\hline D131-8906 & 7.0 & 0.80 & 0.62 & 7.0 & 0.72 & 1.00 & 0.00 & 7.0 & 0.81 & 0.09 & 0.62 \\
\hline D131-9003 & 8.0 & 0.87 & 0.20 & 8.0 & 0.89 & 0.61 & 0.50 & 8.0 & 0.89 & 0.40 & 0.13 \\
\hline D132-8806 & 15.0 & 0.54 & 1.34 & 14.0 & 0.50 & 0.78 & 0.58 & 14.0 & 0.59 & 0.90 & 0.68 \\
\hline D132-8909 & 5.0 & 0.87 & 0.26 & 5.0 & 0.69 & 0.69 & 0.54 & 5.0 & 0.69 & 0.51 & 0.54 \\
\hline D133-8707 & 33.9 & 0.31 & 2.04 & 29.2 & 0.34 & 0.41 & 1.97 & 23.0 & 0.47 & 1.00 & 0.18 \\
\hline D133-8806 & 69.6 & 0.34 & 3.06 & 54.2 & 0.39 & 0.62 & 1.74 & 134.1 & 0.17 & 0.10 & 2.73 \\
\hline D133-8906 & 31.6 & 0.32 & 2.79 & 22.5 & 0.36 & 0.74 & 1.11 & 24.2 & 0.45 & 0.64 & 2.93 \\
\hline D133-8909 & 24.5 & 0.51 & 1.54 & 23.3 & 0.51 & 0.60 & 1.48 & 22.0 & 0.53 & 0.94 & 0.36 \\
\hline D134-8906 & 13.0 & 0.87 & 0.31 & 13.0 & 0.88 & 0.68 & 0.82 & 13.0 & 0.84 & 0.35 & 0.35 \\
\hline D134-9003 & 8.0 & 0.67 & 0.44 & 8.0 & 0.63 & 0.74 & 0.35 & 8.0 & 0.64 & 1.00 & 0.01 \\
\hline G062-8806 & 14.3 & 0.50 & 1.50 & 13.0 & 0.45 & 0.77 & 0.58 & 13.0 & 0.51 & 0.90 & 0.88 \\
\hline G06G-8707 & 35.7 & 0.48 & 4.46 & 119.0 & 0.16 & 0.06 & 3.30 & 31.0 & 0.51 & 0.92 & 2.06 \\
\hline G06G-8806 & 279.8 & 0.01 & 2.52 & 13.3 & 0.15 & 0.43 & 1.74 & 10.0 & 0.30 & 0.97 & 1.12 \\
\hline G06G-8909 & 7.1 & 0.82 & 2.16 & 7.0 & 0.89 & 0.45 & 1.42 & 7.0 & 0.66 & 0.72 & 2.11 \\
\hline H131-8906 & 17.4 & 0.62 & 1.53 & 17.7 & 0.62 & 0.58 & 1.52 & 17.0 & 0.68 & 0.93 & 0.70 \\
\hline H131-8909 & 9.0 & 0.69 & 0.39 & 9.0 & 0.67 & 0.75 & 0.34 & 9.0 & 0.68 & 0.82 & 0.21 \\
\hline $\mathrm{H} 131-9003$ & 12.8 & 0.54 & 2.33 & 12.0 & 0.42 & 0.87 & 0.14 & 27.7 & 0.22 & 0.06 & 2.14 \\
\hline H132-8906 & 30.0 & 0.26 & 2.53 & 39.7 & 0.20 & 0.17 & 2.50 & 18.0 & 0.39 & 0.96 & 0.36 \\
\hline H133-8906 & 56.8 & 0.21 & 2.52 & 60.4 & 0.20 & 0.19 & 2.51 & 29.0 & 0.40 & 0.99 & 0.31 \\
\hline H134-8906 & 18.1 & 0.48 & 1.45 & 16.2 & 0.49 & 0.70 & 1.14 & 16.0 & 0.51 & 0.90 & 0.55 \\
\hline
\end{tabular}


Sur ces cinq stations, le modèle 22 ( $P$ c constante sur les deux premiers passages) a été retenu à quatre reprises, le modèle 21 ( $P c$ constante sur les deux derniers passages) ne se révélant le mieux adapté que sur une seule station.

Il est à noter que, dans ces cinq cas, Pc a augmenté au cours des opérations. Les estimations réalisées sont par conséquent plus faibles (environ de $20 \%$ ) que celles qui auraient été obtenues avec le modèle classique à Pc constante.

\section{Variation de la probabilité de capture en fonction du nombre de passages considérés}

Dans $21 \%$ des cas ( 6 stations), les Pc les plus probables au premier passage, calculées respectivement sur les deux premiers passages, et sur l'ensemble des trois passages ne sont pas différentes et conduisent donc à la même estimation des effectifs présents dans les stations concernées (tableaux II et III).

Dans une station sur deux, cette même Pc calculée sur les deux premiers passages est plus forte et conduit à des sous-estimations des effectifs les plus probables. II est d'ailleurs à noter que, dans 9 cas (1/3 des stations), l'estimation calculée à partir des deux premiers passages s'est révélée plus faible que la somme des captures effectivement réalisées au terme des trois passages (tableau I).

Enfin, dans $29 \%$ des cas ( 7 stations), Pc au premier passage calculée sur les deux premiers passages est plus faible que celle calculée sur l'ensemble des trois passages et conduit donc à une surestimation des effectifs présents dans les stations concernées.

\section{DISCUSSION}

\section{Effet «taille»}

Sur notre jeu de données émanant de 6 campagnes différentes d'inventaires en canaux et fossés en marais d'eau douce, aucun effet "taille" n'a pu être mis en évidence sur la probabilité de capture de l'anguille en pêche électrique.

Cette conclusion va à l'encontre des travaux de RANDALL (1990) qui montrent que la probabilité de capture des saumons atlantiques étaient plus faibles pour les smolts que pour les parrs. De même, KLEIN BRETELER et al. (1990) mettent en évidence une diminution de l'efficacité de la pêche électrique pour les petites classes de taille de rotengle, de tanche, de perche et de sandre ; ce phénomène ne pouvant toutefois être généralisé à l'ensemble des plans d'eau qu'ils avaient étudiés. Le même type de conclusion a été avancé par BÜTTIKER (1992) sur Salmo trutta.

LIBOSVARSKY (1966) par contre, au terme d'un travail sur la même espèce, parvenait dans la très grande majorité des cas aux conclusions inverses.

En ce qui concerne l'anguille, les résultats publiés sont également contradictoires (NAISMITH et KNIGHTS, 1990 ; ZALEWSKI et COWX, 1990 ; CALLAGHAN et McCARTHY, 1992).

Dans le cas de l'anguille dans les marais de Bourgneuf, la recommandation de nombreux auteurs (BOHLIN et SUNDSTRÖM, 1977 ; MAHON et al., 1979 ; PHILLIPPART, 1979 ; MAHON, 1980 ; COWX, 1983) d'effectuer les estimations d'abondance par classe de taille ne semble pas devoir être retenue. Une estimation toutes classes de taille confondues a de plus l'avantage de considérer un plus grand nombre d'individus capturés pour chaque calcul et donc d'augmenter la fiabilité de l'estimation (ZIPPIN, 1956).

L'absence de variation significative de $\mathrm{Pc}$ en fonction de la taille des individus, en considérant les probabilités obtenues dans chaque classe de taille, doit toutefois être nuancée. En effet, une variabilité significative entre les stations a été mise en évidence.

Cet effet "station", très vraisemblablement lié aux caractéristiques environnementales (ici, niveau du colmatage et de la colonisation végétale) de chaque site inventorié, rarement pris en compte, pourrait en effet venir masquer un effet “taille». 
Ceci permettrait d'expliquer tout ou partie des résultats très contradictoires affichés jusqu'à présent par les divers auteurs sur de nombreuses espèces. En ce qui concerne nos données en marais, cette analyse est actuellement en cours.

\section{Nombre de passages}

Les différences significatives enregistrées entre les estimations à partir de 2 ou 3 passages (différence moyenne de $20 \%$ ) et la mise en évidence, dans un pourcentage non négligeable de stations, d'une augmentation de la probabilité de capture après le deuxième passage, montrent l'intérêt d'un troisième passage dans une optique d'estimation quantitative du nombre le plus probable d'anguilles présentes dans une station donnée.

On peut d'ailleurs remarquer que, dans un tiers des stations, on a la certitude que l'estimation sur 2 passages est effectivement erronée puisqu'elle est plus faible que la somme des captures obtenues sur 3 passages.

II est également à signaler que les essais d'application du modèle 21 aux seuls deux premiers passages n'ont pas abouti dans un cas, le point de blocage n'ayant pas encore été identifié.

Parmi les 28 stations, en considérant les trois passages et les captures correspondantes d'anguilles toutes tailles confondues, un modèle classique à Pc constante semble approprié dans plus de $80 \%$. En effet, l'utilisation d'un modèle à Pc variable d'un passage à l'autre ne s'impose que dans cinq des stations de notre échantillon.

Dans ces 5 cas, l'utilisation d'un modèle classique à Pc constante conduit à surestimer (mini $8 \%$, maxi $93 \%$, moyenne $40 \%$ ) l'effectif le plus probable des animaux présents. Ceci ne rejoint pas les conclusions les plus couramment énoncées sur d'autres espèces, conclusions évoquant une sous-estimation des effectifs liée à une diminution de la probabilité de capture lors des passages successifs (BOHLIN, 1982 ; BOHLIN et COWX, 1990).

Cette augmentation de Pc observée sur ces 5 stations, le plus souvent après le deuxième passage, tend à accréditer la thèse de "déstabilisation" des individus"par les passages précédents proposée par SCHNUTE en 1983. Elle peut également révéler une amélioration de l'efficacité de l'équipe de pêche liée au nombre plus faible de poissons à capturer.

\section{CONCLUSION}

Le traitement des données obtenues sur l'anguille lors d'inventaires en marais d'eau douce nous a permis de préciser certains points importants à prendre en compte dans notre approche de l'espèce dans ces milieux peu étudiés jusqu'à maintenant.

La pêche électrique semble, en effet, pouvoir être un outil de quantification intéressant pour peu que certaines règles soient respectées.

L'attention portée lors de ces opérations au maintien de la constance de l'effort de pêche lors des passages successifs, respect obligé dans le cadre d'une prospection par points, doit tout d'abord être rappelée car elle est la condition de base à toute possibilité d'utilisation ultérieure des résultats à des fins d'estimation quantitative.

Sur le plan du traitement des données, l'usage des possibilités offertes par la méthode de Schnute, méthode permettant d'adapter, a posteriori et avec test statistique à l'appui, une évolution de la probabilité de capture lors des différents passages, nous a permis de mettre en évidence le caractère inadapté des modèles classiques (probabilité constante) dans près de $20 \%$ de nos stations.

L'augmentation de la probabilité de capture alors observée dans tous les cas problématiques conduit à une révision notable, à la baisse des estimations effectuées avec les modèles à probabilité constante. 
Au vu des résultats obtenus, il semble hautement souhaitable, dans une démarche de quantification sur une station donnée, de réaliser au moins trois passages successifs. Ceci étant, la nécessaire prise en compte du temps et des moyens disponibles pour réaliser un nombre significatif de stations dans un territoire donné lors de chaque campagne conduira à se limiter, dans une grande majorité des cas, à la réalisation de trois passages.

Le traitement sur l'ensemble des stations inventoriées ne permet pas de révéler un effet " taille" sur l'évolution des probabilités de capture. Cependant, une analyse menée à l'échelle de la station doit être menée afin de préciser une possible interférence entre les effets " taille des individus " et " caractéristiques de la station de pêche".

De ces considérations de base découleront à terme les possibilités d'utilisation de la pêche électrique :

- soit pour une quantification des stocks d'anguilles présents dans divers habitats du marais à partir d'inventaires à plusieurs passages sur secteurs isolés,

- soit pour une approche par indicateurs (présence/absence, indices d'abondance) obtenus par passage unique sur un grand nombre de stations (FEUNTEUN, 1994) en s'inspirant de la démarche initiée par NELVA (1979) sur les grands fleuves.

Obtenues dans ces marais littoraux aux caractéristiques bien particulières, il serait bien sûr intéressant de déterminer dans quelle mesure ces conclusions sont généralisables aux cours d'eau plus classiques, pour peu que les opérations y aient été effectuées dans le même esprit.

\section{BIBLIOGRAPHIE}

ANONYME, 1984. Rapport de synthèse et programme quinquennal. Groupe national anguille, $60 \mathrm{p}$.

BACKIEL T., 1980. Introduction. In "Guidelines for Sampling Fish in Inland Waters". EIFAC Techn. Paper 33.

BOHLIN T., 1982. The validity of the removal method for small populations - Consequences for electrofishing practice. Rep. Inst. Freshwat. Res. Drottningholm, 60, 15-18.

BOHLIN T. and SUNDSTRÖM B., 1977. Influence of unequal catchability on population estimates using the Lincoln index and the removal method applied to ellecto-fishing. Oikos, 28, 123-129.

BOHLIN T. and COWX I.G., 1990. Implications of unequal probability of capture by fishing on the estimation of population size. in Cowx I.G., Developments in electric fishing, 145-155, Fishing news books.

CALLAGHAN R.M. and MCCARTHY T.Kieran, 1992. Variations in population structure ang growth rate of eels in the Dunkellin river system, wertern Ireland. Ir. Fish. Invest. (A. Freshwat.), 36, 59-65.

CARLE F.L. and STRUB M.R., 1978. A new method for estimating population size from removal data. Biometrics, 34, 621-630.

COWX I.G., 1983. Review of the methods for estimating fish population size from survey removal data. Fish. Mgmt, 14, $n^{\circ} 2,67-82$.

DE LURY D.B., 1947. On the estimation of biological population. Biometrics, 3, 145-147.

ELIE P. et RIGAUD C.,1984. Etude de la population d'anguilles de l'estuaire et du bassin versant de la Vilaine : examen particulier de l'impact du barrage d'arzal sur la migration anadrome (civelles) - propositions d'amélioration du franchissement de cet obstacle. Faculté des sciences de Rennes - CEMAGREF de Bordeaux, 192 p. 
FEUNTEUN E., 1987. Contribution à l'étude du peuplement ichtyologique du marais de Bourgneuf en Retz. Rapport de DEA Biologie des populations et éco-éthologie. Univ de Rennes I - Cemagref Bordeaux, 53p + annexes.

FEUNTEUN E., 1994. Le peuplement pisicole du marais littoral endigué de BourgneufMachecoul (France Loire-Atlantique). Thèse de doctorat en sciences biologiques. Université de Rennes I, $240 \mathrm{p}$.

FEUNTEUN E., RIGAUD C., ELIE P., LEFEUVRE J.B., 1992. Les marais doux endigués de Bourgneuf-Machecoul (Pays de Loire). Premiers éléments de connaissances du peuplement piscicole- Relation ichtyofaune-habitat et problèmes majeurs de gestion. Rev. Fr. Sci. Eau, 5 (4), 509-527.

GERDEAUX D., 1987. Revue des méthodes d'estimation de l'effectif d'une population par pêches successives avec retrait - Programme d'estimation d'effectif par la méthode de Carle et Strub. Bull. Fr. Pêche Piscic., 304, 13-21.

LAMARQUE P., ARRIGNON J. et GOSSET C., 1978. Comparaison des appareils de pêche à l'électricité EPMC et Héron. Bull. Fr. Pêche Piscic., 270, 223-236.

LAMBERT P., 1994. Mise au point d'une technique d'inventaire de l'anguille par pêche électrique. Exploitation des données CSP et CEMAGREF. Rapport d'étude CSPCEMAGREF, $32 \mathrm{p}$

LEGAULT A., 1987. L'anguille dans le bassin de la Sèvre Niortaise Biologie Ecologie exploitation. Publications du Département d'halieutique $n^{\circ} 6$ - ENSA Rennes, $305 \mathrm{p}$.

LIBOSVARSKY J., 1990. Notes on electrofishing mainly on the probabilty of capture. In Cowx I.G., Developments in electric fishing, 140-144, Fishing news books.

MAHON R., 1980. Accuracy of cath-effort methods for estimating density and biomass in streams. Env. Biol. Fish., 5 (4), 343-360.

MAHON R., BALON U.K., and NOAKES D.L.G., 1979. Distribution, community structure and production of fishes in the upper Speed River, Ontario : a preimpoundment study. Env. Biol. Fish., 4 (3), 219-244.

MORAN P.A.P., 1951. A mathematical theory of animal trapping. Biometrika, 38, 307-311.

NAISMITH I.A. and KNIGHTS B., 1990. Studies of fyke netting, electrofishing and other sampling methods and of techniques for estimating populations of eels, Anguilla anguilla L.. Aquac. and Fish Managt, 21(3), 357-363.

NELVA A., PERSAT H., CHESSEL D., 1979. Une nouvelle méthode d'étude des peuplements ichtyologiques dans les grands cours d'eau par échantillonnage ponctuel d'abondance. C.R. Acad. Sci., Paris. 289(D), 1295-1298.

PHILLIPART J.C., 1979. Observations concernant l'efficacité de la pêche à l'électricité dans une zone à barbeau. Bull. Fr. Pêche Piscic., 273, 157-171.

RANDALL R.C., 1990. Effect of water temperature, depth, conductivity and survey area on the catchability of juvenile Atlantic salmon by electric fishing in New Brunswick streams. in Cowx I.G., Developments in electric fishing, 79-90, Fishing news books.

SCHNUTE J., 1983. A new approach to estimating population by the removal method. Can. J. Fish. Sci., vol 40, 2153-2169.

ZALEWSKI M., COWX I.G., 1990. Factors affecting the efficiency of electric fishing. In Fishing with Electricity (Cowx \& Lamarque Eds). Oxford : Fishing News Books.

ZIPPIN C., 1956. An evaluation of the removal method of estimating animal populations. Biometrics, 12, 163-189. 